\title{
Inequalities for $\log$-convex functions via three times differentiability
}

\author{
Merve Avcı Ardıç ${ }^{1 *}$, M. Emin Özdemir ${ }^{2}$ \\ ${ }^{1}$ Adiyaman University, Faculty of Science and Arts, Department of Mathematics, Adiyaman, Turkey, \\ merveavci@gmail.com \\ 2 Uludağ University, Education Faculty, Bursa, Turkey, \\ eminozdemir@uludag.edu.tr \\ ${ }^{*}$ Corresponding Author \\ Recieved: $28^{\text {th }}$ October 2016 \\ Accepted: $30^{\text {th }}$ December 2016 \\ DOI:10.18466/cbayarfbe.319873
}

\begin{abstract}
In this paper, some new integral inequalities like Hermite-Hadamard type for functions whose third derivatives absolute value are $\log$-convex are established. Some applications to quadrature formula for midpoint error estimate are given.

Keywords- Convexity, log - convex functions, Hermite-Hadamard inequality, Hölder integral inequality, Power-mean integral inequality
\end{abstract}

\section{Introduction}

We shall recall the definitions of convex functions and $\log$-convex functions:

Let $I$ be an interval in $\mathbb{R}$. Then $f: I \rightarrow \mathbb{R}$ is said to be convex if for all $x, y \in I$ and all $\alpha \in[0,1]$,

$f(\alpha x+(1-\alpha) y) \leq \alpha f(x)+(1-\alpha) f(y)(1.1)$

holds. If (1.1) is strict for all $x \neq y$ and $\alpha \in(0,1)$,

then $f$ is said to be strictly convex. If the inequality in (1.1) is reversed, then $f$ is said to be concave. If it is strict for all $x \neq y$ and $\alpha \in(0,1)$, then $f$ is said to be strictly concave.

A function is called log-convex or multiplicatively convex on a real interval $I=[a, b]$, if $\log f$ is convex, or, equivalently if for all $x, y \in I$ and all $\alpha \in[0,1]$,

$$
f(\alpha x+(1-\alpha) y) \leq f(x)^{\alpha} \cdot f(y)^{(1-\alpha)} .
$$

It is said to be log-concave if the inequality in (1.2) is reversed. For some results for $\log -$ convex functions see $[1,2,3,4,5,6,7]$.

The following inequality is called HermiteHadamard inequality for convex functions: Let $f: I \rightarrow \mathbb{R}$ be a convex function on the interval $I$ of real numbers and $a, b \in I$ with $a<b$. Then double inequality

$$
f\left(\frac{a+b}{2}\right) \leq \frac{1}{b-a} \int_{a}^{b} f(x) d x \leq \frac{f(a)+f(b)}{2}
$$

holds.

The main purpose of this paper is to obtain some new integral inequalities like Hermite-Hadamard type for functions whose third derivatives absolute value are $\log$-convex.

In order to prove our main results for $\log -$ convex functios we need the following Lemma from [8]:

Lemma 1.1. Let $f: I \subset \mathbb{R} \rightarrow \mathbb{R}$ be a three times differentiable mapping on $I^{\circ}$ (the interior of $I)$ and $a, b \in I^{\circ}$ with $a<b$. If $f^{(3)} \in L_{1}[a, b]$, then

$$
\frac{1}{b-a} \int_{a}^{b} f(x) d x-f\left(\frac{a+b}{2}\right)-\frac{(b-a)^{2}}{24} f^{\prime \prime}\left(\frac{a+b}{2}\right)
$$

$=\frac{(b-a)^{3}}{96}\left[\int_{0}^{1} t^{3} f^{(3)}\left(\frac{t}{2} a+\frac{2-t}{2} b\right) d t\right.$

$\left.-\int_{0}^{1} t^{3} f^{(3)}\left(\frac{2-t}{2} a+\frac{t}{2} b\right) d t\right]$.

In the sequel of paper, we deduce

$L_{p}[a, b]=\left\{f:\left(\int_{a}^{b}|f(x)|^{p} d x\right)^{\frac{1}{p}}<\infty, 1 \leq p<\infty\right\}$ 
where $[a, b]$ is a closed interval.

\section{Inequalities for log-convex functions}

We shall start the following result:

Theorem 2.1. Let $f: I \rightarrow[0, \infty)$, be a three times differentiable mapping on $I^{\circ}$ such that $f^{\prime \prime \prime} \in L_{1}[a, b]$ where $a, b \in I^{\circ}$ with $a<b$. If $\left|f^{\prime \prime \prime}\right|$ is $\log$-convex on $[a, b]$, then the following inequality holds:

$\left|\frac{1}{b-a} \int_{a}^{b} f(x) d x-f\left(\frac{a+b}{2}\right)-\frac{(b-a)^{2}}{24} f^{\prime \prime}\left(\frac{a+b}{2}\right)\right|$

$\leq \frac{(b-a)^{3}}{96}\left\{\left|f^{\prime \prime \prime}(b)\right| \mu_{K}+\left|f^{\prime \prime \prime}(a)\right| \mu_{M}\right\}$

where

$\mu_{K}=\frac{2 K^{\frac{1}{2}}(\ln K-6)}{(\ln K)^{2}}+\frac{48 K^{\frac{1}{2}}(\ln K-2)}{(\ln K)^{4}}+\frac{96}{(\ln K)^{4}}$,

$\mu_{M}=\frac{2 M^{\frac{1}{2}}(\ln M-6)}{(\ln M)^{2}}+\frac{48 M^{\frac{1}{2}}(\ln M-2)}{(\ln M)^{4}}+\frac{96}{(\ln M)^{4}}$

$$
\begin{aligned}
& =\frac{(b-a)^{3}}{96}\left\{\left|f^{\prime \prime \prime}(b)\right| \int_{0}^{1} t^{3}\left|\frac{\left\lceil f^{\prime \prime \prime}(a) \mid\right\rceil^{\frac{t}{2}}}{\left|f^{\prime \prime \prime}(b)\right|}\right|^{d} d t\right. \\
& \left.\left|f^{\prime \prime \prime}(a)\right| \int_{0}^{1} t^{3}\left|\frac{\left|f^{\prime \prime \prime}(b)\right|}{\left|f^{\prime \prime \prime}(a)\right|}\right|^{\frac{t}{2}} d t\right\} .
\end{aligned}
$$

The proof is completed by making use of the neccessary computation.

Corollary 2.1. Let $\mu_{K}, \mu_{M}, K$ and $M$ be defined as in Theorem 2.1. If we choose $f^{\prime \prime}\left(\frac{a+b}{2}\right)=0$ in Theorem 2.1, we obtain the following inequality

$$
\begin{aligned}
& \left|\frac{1}{b-a} \int_{a}^{b} f(x) d x-f\left(\frac{a+b}{2}\right)\right| \\
\leq & \frac{(b-a)^{3}}{96}\left\{f^{\prime \prime \prime}(b)\left|\mu_{K}+\right| f^{\prime \prime \prime}(a) \mid \mu_{M}\right\} .
\end{aligned}
$$

and

$$
K=\frac{\left|f^{\prime \prime \prime}(a)\right|}{\left|f^{\prime \prime \prime}(b)\right|}, M=\frac{\left|f^{\prime \prime \prime}(b)\right|}{\left|f^{\prime \prime \prime}(a)\right|} .
$$

In the sequel of the paper, we set $K, M \neq 1$.

Proof. From Lemma 1.1, property of the modulus and $\log$-convexity of $\left|f^{\prime \prime \prime}\right|$ we have

$$
\begin{aligned}
& \left|\frac{1}{b-a} \int_{a}^{b} f(x) d x-f\left(\frac{a+b}{2}\right)-\frac{(b-a)^{2}}{24} f^{\prime \prime}\left(\frac{a+b}{2}\right)\right| \\
& \leq \frac{(b-a)^{3}}{96}\left\{\int_{0}^{1} t^{3}\left|f^{\prime \prime \prime}\left(\frac{t}{2} a+\frac{2-t}{2} b\right)\right| d t\right. \\
& \left.+\int_{0}^{1} t^{3}\left|f^{\prime \prime \prime}\left(\frac{t}{2} b+\frac{2-t}{2} a\right)\right| d t\right\}
\end{aligned}
$$

Theorem 2.2. Let $f: I \rightarrow[0, \infty)$, be a three times differentiable mapping on $I^{\circ}$ such that $f^{\prime \prime} \in L_{1}[a, b]$ where $a, b \in I^{\circ}$ with $a<b$. If $\left|f^{\prime \prime \prime}\right|$ is $\log$-convex on $[a, b]$, then the following inequality holds for some fixed $q>1$

$\left|\frac{1}{b-a} \int_{a}^{b} f(x) d x-f\left(\frac{a+b}{2}\right)-\frac{(b-a)^{2}}{24} f^{\prime \prime}\left(\frac{a+b}{2}\right)\right|$

$$
\begin{aligned}
& \leq \frac{(b-a)^{3}}{96}\left(\frac{1}{3 p+1}\right)^{\frac{1}{p}}\left\{\left|f^{\prime \prime \prime}(b)\right|\left(\frac{2}{q \ln K}\left[K^{\frac{q}{2}}-1\right]\right)^{\frac{1}{q}}\right. \\
& \left.+\mid f^{\prime \prime \prime}(a)\left(\frac{2}{q \ln M}\left[M^{\frac{q}{2}}-1\right\rceil\right)^{\frac{1}{q}}\right\}
\end{aligned}
$$

where $K$ and $M$ are as in Theorem 2.1. and 
$\frac{1}{p}+\frac{1}{q}=1$.

Proof. From Lemma 1.1 and using the Hölder integral inequality, we obtain

$$
\begin{aligned}
& \left|\frac{1}{b-a} \int_{a}^{b} f(x) d x-f\left(\frac{a+b}{2}\right)-\frac{(b-a)^{2}}{24} f^{\prime \prime}\left(\frac{a+b}{2}\right)\right| \leq \frac{(b-a)^{3}}{96}\left(\frac{1}{3 p+1}\right)^{\frac{1}{p}}\left\{\left.\left|f^{\prime \prime \prime}(b)\right| \frac{2}{q \ln K}\left|K^{\frac{q}{2}}-1\right|\right|^{\frac{1}{q}}\right. \\
& \leq \frac{(b-a)^{3}}{96}\left\{\left(\int_{0}^{1} t^{3 p} d t\right)^{\frac{1}{p}}\left(\int_{0}^{1}\left|f^{\prime \prime \prime}\left(\frac{t}{2} a+\frac{2-t}{2} b\right)\right|^{q} d t\right)^{\frac{1}{q}}+\left|f^{\prime \prime \prime}(a)\right|\left(\frac{2}{q \ln M}\left[M^{\frac{q}{2}}-\left.1\right|^{\frac{1}{q}}\right\}\right.\right. \\
& \left.+\left(\int_{0}^{1} t^{3 p} d t\right)^{\frac{1}{p}}\left(\int_{0}^{1}\left|f^{\prime \prime \prime}\left(\frac{t}{2} b+\frac{2-t}{2} a\right)\right|^{q} d t\right)^{\frac{1}{q}}\right\} . \quad \text { where } q>1, \frac{1}{p}+\frac{1}{q}=1 .
\end{aligned}
$$

Since $\left|f^{\prime \prime}\right|$ is $\log$-convex on $[a, b]$ we can say $\left|f^{\prime \prime}\right|^{q}$ is also $\log -$ convex on $[a, b]$. If we use the $\log$-convexity of $\left|f^{\prime \prime}\right|^{q}$ above, we can write

$\left|\frac{1}{b-a} \int_{a}^{b} f(x) d x-f\left(\frac{a+b}{2}\right)-\frac{(b-a)^{2}}{24} f^{\prime \prime}\left(\frac{a+b}{2}\right)\right|$

$\leq \frac{(b-a)^{3}}{96}\left\{\left(\int_{0}^{1} t^{3 p} d t\right)^{\frac{1}{p}}\left(\int_{0}^{1}\left|f^{\prime \prime \prime}(a)\right|^{\frac{q t}{2}}\left|f^{\prime \prime \prime}(b)\right|^{q-\frac{q t}{2}} d t\right)^{\frac{1}{q}}\right.$

$\left.+\left(\int_{0}^{1} t^{3 p} d t\right)^{\frac{1}{p}}\left(\int_{0}^{1}\left|f^{\prime \prime \prime}(b)\right|^{\frac{q t}{2}}\left|f^{\prime \prime \prime}(a)\right|^{q-\frac{q t}{2}} d t\right)^{\frac{1}{q}}\right\}$

$=\frac{(b-a)^{3}}{96}\left(\frac{1}{3 p+1}\right)^{\frac{1}{p}}\left\{\mid f^{\prime \prime \prime}(b)\left(\frac{2}{q \ln K}\left[K^{\frac{q}{2}}-1\right]\right)^{\frac{1}{q}}\right.$

$\left.+\mid f^{\prime \prime \prime}(a)\left(\frac{2}{q \ln M}\left[M^{\frac{q}{2}}-1\right]\right)^{\frac{1}{q}}\right\}$.

The proof is completed.

Corollary 2.2. Let $K$ and $M$ be defined as in

Theorem 2.2. If we choose $f^{\prime \prime}\left(\frac{a+b}{2}\right)=0$ in
Theorem 2.2, we obtain the following inequality

$\left|\frac{1}{b-a} \int_{a}^{b} f(x) d x-f\left(\frac{a+b}{2}\right)\right|$

Theorem 2.3. Let $f: I \rightarrow[0, \infty)$, be a three times differentiable mapping on $I^{\circ}$ such that $f^{\prime \prime \prime} \in L_{1}[a, b]$ where $a, b \in I^{\circ}$ with $a<b$. If $\left|f^{\prime \prime \prime}\right| \quad$ is $\log -$ convex on $[a, b]$. Then the following inequality holds for some fixed $q \geq 1$ :

$\left|\frac{1}{b-a} \int_{a}^{b} f(x) d x-f\left(\frac{a+b}{2}\right)-\frac{(b-a)^{2}}{24} f^{\prime \prime}\left(\frac{a+b}{2}\right)\right|$

$\leq \frac{(b-a)^{3}}{96}\left(\frac{1}{4}\right)^{1-\frac{1}{q}}\left\{\left|f^{\prime \prime \prime}(b)\right|\left(\mu_{K, q}\right)^{\frac{1}{q}}+\left|f^{\prime \prime \prime}(a)\right|\left(\mu_{M, q}\right)^{\frac{1}{q}}\right\}$
where

$$
\begin{aligned}
& \mu_{K, q}=\frac{2 K^{\frac{q}{2}}(q \ln K-6)}{(q \ln K)^{2}}+\frac{48 K^{\frac{q}{2}}(q \ln K-2)}{(q \ln K)^{4}} \\
& +\frac{96}{(q \ln K)^{4}}, \\
& \mu_{M, q}=\frac{2 M^{\frac{q}{2}}(q \ln M-6)}{(q \ln M)^{2}}+\frac{48 M^{\frac{q}{2}}(q \ln M-2)}{(q \ln M)^{4}} \\
& +\frac{96}{(q \ln M)^{4}}
\end{aligned}
$$

and $K, M$ are as in Theorem 2.1.

Proof. From Lemma 1.1, using the well-known power-mean integral inequality and $\log$-convexity of $\left|f^{\prime \prime \prime}\right|^{q}$ we have

$\left|\frac{1}{b-a} \int_{a}^{b} f(x) d x-f\left(\frac{a+b}{2}\right)-\frac{(b-a)^{2}}{24} f^{\prime \prime}\left(\frac{a+b}{2}\right)\right|$ 


$$
\begin{aligned}
& \leq \frac{(b-a)^{3}}{96}\left\{\left(\int_{0}^{1} t^{3} d t\right)^{1-\frac{1}{q}}\left(\int_{0}^{1} t^{3}\left|f^{\prime \prime \prime}\left(\frac{t}{2} a+\frac{2-t}{2} b\right)\right|^{q} d t\right)^{\frac{1}{q}}\right. \\
& \left.+\left(\int_{0}^{1} t^{3} d t\right)^{1-\frac{1}{q}}\left(\int_{0}^{1} t^{3}\left|f^{\prime \prime \prime}\left(\frac{t}{2} b+\frac{2-t}{2} a\right)\right|^{q} d t\right)^{\frac{1}{q}}\right\} \\
& \leq \frac{(b-a)^{3}}{96}\left\{\left(\int_{0}^{1} t^{3} d t\right)^{1-\frac{1}{q}}\left(\int_{0}^{1} t^{3}\left|f^{\prime \prime \prime}(a)\right|^{\frac{q t}{2}}\left|f^{\prime \prime \prime}(b)\right|^{q-\frac{q t}{2}} d t\right)^{\frac{1}{q}}\right. \\
& \left.+\left(\int_{0}^{1} t^{3} d t\right)^{1-\frac{1}{q}}\left(\int_{0}^{1} t^{3}\left|f^{\prime \prime \prime}(b)\right|^{\frac{q t}{2}}\left|f^{\prime \prime \prime}(a)\right|^{q-\frac{q t}{2}} d t\right)^{\frac{1}{q}}\right\} . \\
& \begin{array}{c}
\chi_{2}=\frac{(b-a)^{3}}{96}\left(\frac{1}{3 p+1}\right)^{\frac{1}{p}} \\
\times\left\{\mid f^{\prime \prime \prime}(b)\left(\frac{2}{q \ln K}\left[K^{\frac{q}{2}}-1\right]\right)^{\frac{1}{q}}\right. \\
\left.+\left|f^{\prime \prime \prime}(a)\right|\left(\frac{2}{q \ln M}\left[M^{\frac{q}{2}}-1\right]\right)^{\frac{1}{q}}\right\}, \\
\chi_{3}=\frac{(b-a)^{3}}{96}\left(\frac{1}{4}\right)^{1-\frac{1}{q}}\left\{\mid f^{\prime \prime \prime}(b)\left(\frac{2 K^{\frac{q}{2}}(q \ln K-6)}{(q \ln K)^{2}}+\right.\right.
\end{array} \\
& \left.+\frac{48 K^{\frac{q}{2}}(q \ln K-2)}{(q \ln K)^{4}}+\frac{96}{(q \ln K)^{4}}\right)^{\frac{1}{q}}+\left|f^{\prime \prime \prime}(a)\right| \\
& \times\left(\frac{2 M^{\frac{1}{2}}(\ln M-6)}{(\ln M)^{2}}+\frac{48 M^{\frac{1}{2}}(\ln M-2)}{(\ln M)^{4}}\right. \\
& \left.\left.+\frac{96}{(\ln M)^{4}}\right)^{\frac{1}{q}}\right\}
\end{aligned}
$$

$$
\begin{gathered}
\left|\frac{1}{b-a} \int_{a}^{b} f(x) d x-f\left(\frac{a+b}{2}\right)\right| \\
\leq \frac{(b-a)^{3}}{96}\left(\frac{1}{4}\right)^{1-\frac{1}{q}}\left\{\left|f^{\prime \prime \prime}(b)\right|\left(\mu_{K, q}\right)^{\frac{1}{q}}+\left|f^{\prime \prime \prime}(a)\right|\left(\mu_{M, q}\right)^{\frac{1}{q}}\right\} .
\end{gathered}
$$

and $K, M$ are as in Theorem 2.1.

Remark 2.1. In Theorem 2.3 and Corollary 2.3, if we choose $q=1$, we obtain Theorem 2.1 and

Corollary 2.4. From Corollaries 2.1-2.3, we have

$$
\left|\frac{1}{b-a} \int_{a}^{b} f(x) d x-f\left(\frac{a+b}{2}\right)\right| \leq \min \left\{\chi_{1}, \chi_{2}, \chi_{3}\right\}
$$

Corollary 2.1 respectively.

where

\section{Applications to midpoint formula}

We give some error estimates to midpoint formula by using the results of Section 2. Let $d$ be a $\chi_{1}=\frac{(b-a)^{3}}{96}\left\{f^{\prime \prime \prime}(b) \mid \frac{2 K^{\frac{1}{2}}(\ln K-6)}{(\ln K)^{2}}+\frac{48 K^{\frac{1}{2}}(\ln K \text { in Retval }[a, b] \text { and consider the formula }}{(\ln K)^{4} \int_{a}^{b} f(x) d x=M(f, d)+E(f, d)}\right.$

$$
\begin{aligned}
+\frac{96}{(\ln K)^{4}}+\left|f^{\prime \prime \prime}(a)\right| \frac{2 M^{\frac{1}{2}}(\ln M-6)}{(\ln M)^{2}} & \text { where } M(f, d)=\sum_{i=0}^{n-1} f\left(\frac{x_{i}+x_{i+1}}{2}\right)\left(x_{i+1}-x_{i}\right) \text { for } \\
& \text { the midpoint version and } E(f, d) \text { denotes the } \\
& \text { associated approximation error. }
\end{aligned}
$$

Proposition 3.1. Let $f: I \rightarrow[0, \infty)$ be a three times differentiable mapping on $I^{\circ}$ with $a, b \in I^{\circ}$ 
such that $a<b$. If $\left|f^{\prime \prime \prime}\right|$ is $\log$-convex function with $f^{\prime \prime \prime} \in L_{1}[a, b]$, then for every division $d$ of $[a, b]$, the midpoint error estimate satisfies

$$
\begin{aligned}
& |E(f, d)| \\
& \leq \sum_{i=0}^{n-1} \frac{\left(x_{i+1}-x_{i}\right)^{4}}{96}\left\{f^{\prime \prime \prime}\left(x_{i+1}\right)\left|\mu_{1}+\right| f^{\prime \prime \prime}\left(x_{i}\right) \mid \mu_{2}\right\}
\end{aligned}
$$

where

$$
\begin{gathered}
\mu_{1}=\frac{2 K_{1}^{\frac{1}{2}}\left(\ln K_{1}-6\right)}{\left(\ln K_{1}\right)^{2}}+\frac{48 K_{1}^{\frac{1}{2}}\left(\ln K_{1}-2\right)}{\left(\ln K_{1}\right)^{4}}+\frac{96}{\left(\ln K_{1}\right)^{4}}, \\
\mu_{2}=\frac{2 M_{1}^{\frac{1}{2}}\left(\ln M_{1}-6\right)}{\left(\ln M_{1}\right)^{2}}+\frac{48 M_{1}^{\frac{1}{2}}\left(\ln M_{1}-2\right)}{\left(\ln M_{1}\right)^{4}} \\
+\frac{96}{\left(\ln M_{1}\right)^{4}}
\end{gathered}
$$

and

$$
K_{1}=\frac{\left|f^{\prime \prime \prime}\left(x_{i}\right)\right|}{\left|f^{\prime \prime \prime}\left(x_{i+1}\right)\right|}, M_{1}=\frac{\left|f^{\prime \prime \prime}\left(x_{i+1}\right)\right|}{\left|f^{\prime \prime \prime}\left(x_{i}\right)\right|} .
$$

Also $K_{1}, M_{1} \neq 1$.

Proof. By applying Corollary 2.1 on the subintervals $\left[x_{i}, x_{i+1}\right], \quad(i=0,1, \ldots, n-1)$ of the division $d$ we have

$$
\begin{aligned}
& \left|\frac{1}{x_{i+1}-x_{i}} \int_{x_{i}}^{x_{i+1}} f(x) d x-f\left(\frac{x_{i}+x_{i+1}}{2}\right)\right| \\
\leq & \frac{\left(x_{i+1}-x_{i}\right)^{3}}{96}\left\{f^{\prime \prime \prime}\left(x_{i+1}\right)\left|\mu_{1}+\right| f^{\prime \prime \prime}\left(x_{i}\right) \mid \mu_{2}\right\} .
\end{aligned}
$$

By summing over $i$ from 0 to $n-1$, we can write

$$
\begin{aligned}
& \left|\int_{a}^{b} f(x) d x-M(f, d)\right| \\
& \leq \sum_{i=0}^{n-1} \frac{\left(x_{i+1}-x_{i}\right)^{4}}{96}\left\{\left|f^{\prime \prime \prime}\left(x_{i+1}\right)\right| \mu_{1}+\left|f^{\prime \prime \prime}\left(x_{i}\right)\right| \mu_{2}\right\}
\end{aligned}
$$

which completes the proof.

Proposition 3.2. Let $f: I \rightarrow[0, \infty)$ be a three times differentiable mapping on $I^{\circ}$ with $a, b \in I^{\circ}$ such that $a<b$. If $\left|f^{\prime \prime \prime}\right|^{q}$ is $\log$ - convex function with $f^{\prime \prime} \in L_{1}[a, b]$ for some fixed $q>1$, then for every division $d$ of $[a, b]$, the midpoint error estimate satisfies

$$
|E(f, d)| \leq\left(\frac{1}{3 p+1}\right)^{\frac{1}{p}} \frac{1}{96} \sum_{i=0}^{n-1}\left(x_{i+1}-x_{i}\right)^{4}
$$$$
\times\left\{\left|f^{\prime \prime \prime}\left(x_{i+1}\right)\right|\left(\frac{2}{q \ln K_{1}}\left[K_{1}^{\frac{q}{2}}-1\right]\right)^{\frac{1}{q}}\right.
$$

$$
\left.+\mid f^{\prime \prime \prime}\left(x_{i}\right)\left(\frac{2}{q \ln M_{1}}\left[M_{1}^{\frac{q}{2}}-1\right]\right)^{\frac{1}{q}}\right\}
$$

where $\frac{1}{p}+\frac{1}{q}=1$ and $K_{1}, M_{1}$ are as defined in Proposition 3.1.

Proof. The proof can be maintained by using Corollary 2.2 like Proposition 3.1.

Proposition 3.3. Let $f: I \rightarrow[0, \infty)$ be a three times differentiable mapping on $I^{\circ}$ with $a, b \in I^{\circ}$ such that $a<b$. If $\left|f^{\prime \prime \prime}\right|^{q}$ is $\log$ - convex function with $f^{\prime \prime} \in L_{1}[a, b]$ for some fixed $q \geq 1$, then for every division $d$ of $[a, b]$, the midpoint error estimate satisfies

$$
\begin{aligned}
& |E(f, d)| \leq \frac{1}{96}\left(\frac{1}{4}\right)^{1-\frac{1}{q}} \sum_{i=0}^{n-1}\left(x_{i+1}-x_{i}\right)^{4} \\
& \times\left\{\left|f^{\prime \prime \prime}\left(x_{i+1}\right)\right|\left(\mu_{1, q}\right)^{\frac{1}{q}}+\left|f^{\prime \prime \prime}\left(x_{i}\right)\right|\left(\mu_{2, q}\right)^{\frac{1}{q}}\right\}
\end{aligned}
$$

where

$$
\begin{aligned}
& \mu_{1, q}=\frac{2 K_{1}^{\frac{q}{2}}\left(q \ln K_{1}-6\right)}{\left(q \ln K_{1}\right)^{2}}+\frac{48 K_{1}^{\frac{q}{2}}\left(q \ln K_{1}-2\right)}{\left(q \ln K_{1}\right)^{4}} \\
& +\frac{96}{\left(q \ln K_{1}\right)^{4}}, \\
& \mu_{2, q}=\frac{2 M_{1}^{\frac{q}{2}}\left(q \ln M_{1}-6\right)}{\left(q \ln M_{1}\right)^{2}}+\frac{48 M_{1}^{\frac{q}{2}}\left(q \ln M_{1}-2\right)}{\left(q \ln M_{1}\right)^{4}} \\
& +\frac{96}{\left(q \ln M_{1}\right)^{4}}
\end{aligned}
$$

and $K_{1}, M_{1}$ are as defined in Proposition 3.1.

Proof. The proof can be maintained by using Corollary 2.3 like Proposition 3.1. 


\section{References}

[1] Alomari, M; Darus M. On the Hadamard's inequality for $\log$ - convex functions on the coordinates, Journal of Inequalities and Applications, Volume 2009, Article ID 283147, p 13.

[2] Dragomir, S.S. Some Jensen's Type Inequalities for $\log$-Convex Functions of Selfadjoint Operators in Hilbert Spaces, Bulletin of the Malaysian Mathematical Sciences Society, 2011; 34(3), 445-454.

[3] Niculescu, C.P. The Hermite-Hadamard inequality for $\log -$ convex functions, Nonlinear Analysis, 2012; 75, 662-669.

[4] Pachpatte, B.G.A note on integral inequalities involving two log-convex functions, Mathematical Inequalities \& Applications. 2004; 7(4), 511-515.

[5] Pečarić, J; Rehman, A. U. On logarithmic convexity for power sums and related results, Journal of Inequalities and Applications, vol. 2008, Article ID 389410, 9 p, 2008.

[6] Yang, G.S.; Tseng, K.L, Wang H.T. A note on integral inequalities of Hadamard type for $\log$ - convex and $\log$-concave functions, Taiwanese Journal of Mathematics. 2012; 16(2), 479-496.

[7] Zhanga, X; Jiang, W. Some properties of $\log -$ convex function and applications for the exponential function, Computers and Mathematics with Applications. 2012; 63, 1111-1116.

[8] Shuang, Y; Wang, Y; Qi, F. Some inequalities of Hermite-Hadamard type for functions whose third derivatives are $(\alpha, m)$ - convex, Journal of Computational Analysis and Applications. 2014; 17(2), 272-279. 